(C) (2013) Trans. Tech. Publications, Switzerland

This document is published in:

L. Bolzoni, E.M. Ruiz-Navas, E. Gordo. Processing of Elemental Titanium by Powder Metallurgy Techniques, Materials Science Forum, (2013) Vol. 765, pp. 383-387.

Doi.: http://dx.doi.org/10.4028/www.scientific.net/MSF.765.383 


\title{
Processing of Elemental Titanium by Powder Metallurgy Techniques
}

\author{
L. Bolzoni $^{1,2, a}$, E.M. Ruiz-Navas ${ }^{1, b}$, E. Gordo ${ }^{1, c}$ \\ ${ }^{1}$ Department of Materials Science and Engineering, University Carlos III of Madrid, \\ Avda. de la Universidad, 30, 28911 Leganes, Madrid - Spain \\ ${ }^{2}$ BCAST - Brunel Centre for Advanced Solidification Technology \\ Brunel University, Uxbridge, Middlesex, UB8 3PH, London - UK \\ abolzoni.leandro@gmail.com, bemruiz@ing.uc3m.es, elena.gordo@uc3m.es
}

Keywords: elemental titanium, powder metallurgy, pressing and sintering, hot-pressing

\begin{abstract}
Titanium is characterised by an outstanding combination of properties like high strength, low density, good corrosion resistance and biocompatibility. Nonetheless, titanium widespread employment at industrial level, especially in the automotive industry, has not been achieved yet because of its high extraction and production costs. Consequently, titanium finds applications mainly in high demanding sectors, such as the aerospace industry or to produce biomedical devices, where the final high cost is not the principal issue. The processing of titanium and its alloys by means of powder metallurgy (PM) techniques is claimed to be a suitable way to reduce the fabrication cost of titanium products as well as offering the possibility to design new alloys which are difficult to obtain using the conventional metallurgical route, for example due to segregation of heavy alloying elements. This work deals with the processing of hydride-dehydride elemental titanium powder by means of different PM methods and aims at investigating the processing of near net-shape, chemically-homogeneous and fine-grained titanium-based components. In particular, properties achievable (i.e. relative density, microstructure and mechanical properties) and problems related to the processing of elemental titanium by both the conventional PM route of pressing and sintering and the advance PM method of hot-pressing are presented.
\end{abstract}

\section{Introduction}

Titanium is a relatively new engineering material in comparison to other structural metals such as steel and aluminium since its exploitation at industrial level did not start till eighteen thirties when the German philologist Wilhelm Kroll came out with a successful extraction process. The main product of the Kroll process is porous metallic titanium sponge which is normally purified by distillation (leaching), jackhammered out, crushed and pressed before its melting in a consumable electrode vacuum arc furnace. Nevertheless, the crushed metallic titanium sponge can also be employed as starting material in powder metallurgy (PM) processes, taking advantage that it is already in the form of powder. Many studies were performed in the eighties of the twentieth century by employing titanium sponge powders as raw material and by processing it by means of different powder metallurgy techniques like: (1) pressing and sintering (P\&S) [1-3], (2) hot isostatic pressing (HIP) $[4,5]$ and (3) uniaxial hot-pressing (HP) [6]. Titanium sponge powders are characterised by the presence of chlorides which generate porosity filled with gas during sintering preventing to obtain fully-dense or pores-free titanium products. Moreover, the porosity filled with gas cannot be sealed using advanced techniques, such as a post-processing by HIP, and makes the material virtually unweldable. 
Currently, among the titanium powder production processes, the hydride-dehydride (HDH) processes and plasma atomization are two of the most commonly employed for producing titanium and titanium alloys powders [7]. The HDH route is a milling process applicable for the fabrication of titanium powder which relies on the great affinity of titanium for hydrogen. The material obtained is a powder characterised by an angular morphology which is, therefore, ideal for its processing by means of the conventional powder metallurgy route $(\mathrm{P} \& \mathrm{~S})$. The main advantage of using PM methods is the fact that they are near-net-shape processes with a higher yield of material and almost no need of machining which should reduce the production costs of titanium products.

The aim of this work is to study the processing of near net-shape, chemically-homogeneous and high-density titanium-based components starting from $\mathrm{HDH}$ powder to prevent the problems connected with the employment of sponge titanium powders. Specifically, the HDH powder is consolidated by means of $\mathrm{P} \& \mathrm{~S}$ and uniaxial HP varying the processing temperature to study its influence of the final properties.

\section{Experimental Procedure}

The starting powder is HDH titanium purchased from GfE Gesellschaft für Elektrometallurgie mbH The particle size distribution measured by a laser beam analyser and the chemical analysis (LECO) are summarised in Table 1 whilst the morphology is visible in Figure 1. It can be noticed that it is a powder characterised by an irregular morphology with a particle size smaller than $75 \mu \mathrm{m}$ and a chemical composition which can be classified as commercially-pure (CP) titanium grade 4, being oxygen lower than $0.40 \mathrm{wt} . \%$, nitrogen lower than $0.05 \mathrm{wt} . \%$ and carbon lower than $0.08 \mathrm{wt} . \%$ [8].

Table 1. Particle size distribution and chemical analysis of the HDH elemental titanium powder.

\begin{tabular}{|c|c|c|}
\hline \multicolumn{2}{|c|}{ Property } & Ti HDH \\
\hline \hline \multirow{2}{*}{$\begin{array}{c}\text { Particle size } \\
\text { analysis }\end{array}$} & $\mathbf{D}_{\mathbf{5 0}}[\boldsymbol{\mu m}]$ & 37.59 \\
\cline { 2 - 3 } & $\mathbf{D}_{\max }[\boldsymbol{\mu m}]$ & $<75$ \\
\hline \multirow{4}{*}{$\begin{array}{c}\text { Chemical } \\
\text { analysis }\end{array}$} & $\mathbf{O}[\mathbf{w t}$ \%] & 0.272 \\
\cline { 2 - 3 } & $\mathbf{N}[\mathbf{w t} \%]$ & 0.0159 \\
\cline { 2 - 3 } & $\mathbf{C}[\mathbf{w t} \%]$ & 0.0202 \\
\hline
\end{tabular}

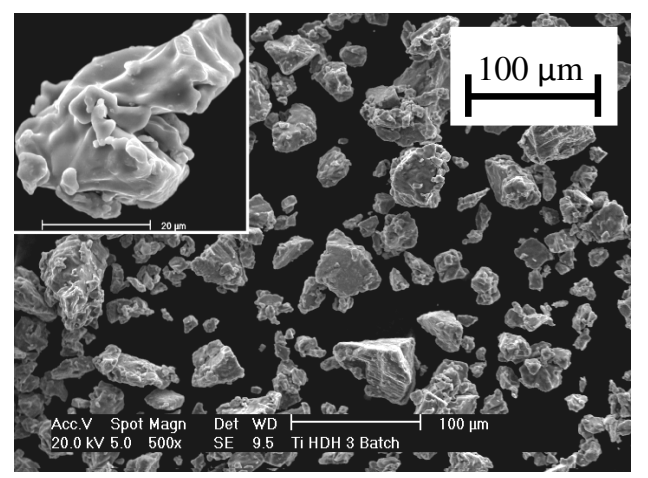

Figure 1. SEM micrograph showing the morphology of the HDH CP-Ti powder.

For the P\&S route, green samples were cold uniaxially pressed at $700 \mathrm{MPa}$ using a floating die and zinc stearate as lubricant for the walls of the die. The sintering of the specimens was carried out in a high vacuum tubular furnace using a heating and cooling rate of $5^{\circ} \mathrm{C} / \mathrm{min}$ and a minimum vacuum level of $10^{-5}$ mbar was guaranteed. The sintering temperature studied ranges between $900^{\circ} \mathrm{C}$ and $1300^{\circ} \mathrm{C}$, step of $200^{\circ} \mathrm{C}$, and the dwell time was fixed at 2 hours.

In the case of the uniaxial HP process, the powder was poured into a graphite mould lined out with a low reactive graphite foil. Graphite foils coated with a high temperature ceramic boron nitride (BN) spray were used to prevent the direct interaction of the powder with the graphite punches at high temperature. The powder was hot-pressed by applying a compaction pressure of $30 \mathrm{MPa}$ under a minimum vacuum level of $10^{-1} \mathrm{mbar}$ and using a heating rate of $10^{\circ} \mathrm{C} / \mathrm{min}$. Three processing temperatures, $900^{\circ} \mathrm{C}, 1100^{\circ} \mathrm{C}$ and $1300^{\circ} \mathrm{C}$, were employed to sinter the powder and an effective dwell time at maximum temperature of 30 minutes was set. 
Prior to characterisation, the uniaxial hot-pressed samples were sandblasted to clean the surfaces that had been in contact with the processing tools. Water displacement measurements based on Archimedes' principle were carried out to measure the density of the sintered samples. The relative density was calculated using the theoretical value of wrought elemental titanium, $4.51 \mathrm{~g} / \mathrm{cm}^{3}$ [8]. The variation of the hardness with the sintering temperature was measured on the cross-section of the samples using a Wilson Wolpert Universal Hardness DIGI-TESTOR 930 tester performing HV30 measurements. The classical metallographic route (grinding with SiC papers, polishing with silica gel and etching using Kroll's reagent) was followed to prepare the samples for the microstructural analysis (Olympus GX71 optical microscope and in a Philips XL-30 SEM).

\section{Results and Discussion}

\subsection{Relative density of sintered materials}

The variation of the relative density versus the processing temperature for $\mathrm{P} \& \mathrm{~S}$ and HP samples is presented in Figure 2 where it can be seen that, independently of the processing technique, the relative density of the specimens increases with the increment of the processing temperature. In the case of the P\&S samples the relative density increases of approximately $3-4 \%$ every $200^{\circ} \mathrm{C}$ whereas in the case of the HP samples there is a slight increment with the processing temperature of almost $0.5 \%$ every $200^{\circ} \mathrm{C}$. Nonetheless, the most important aspect is the fact that the application of a uniaxial pressure during the sintering step is, actually, beneficial to reach high relative density values. This is due to the fact that

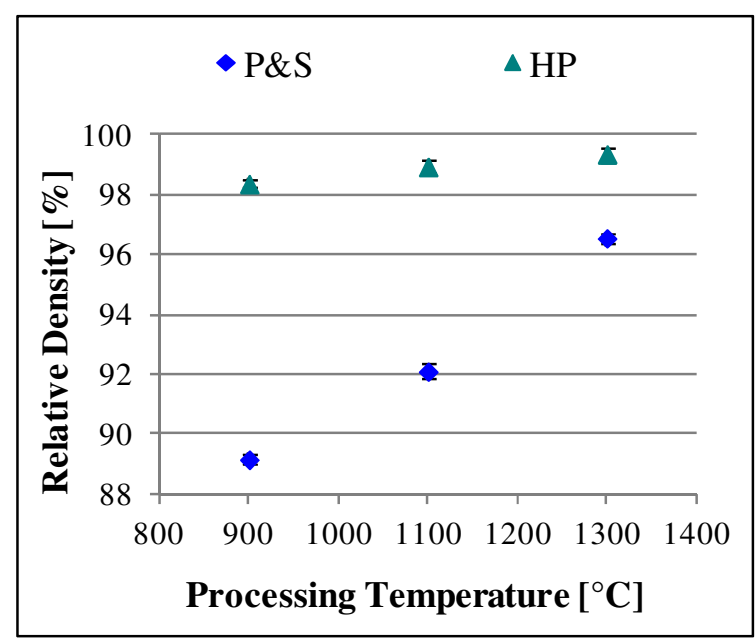

Figure 2. Relative density of P\&S and HP samples as a function of the processing temperature. the processing temperature studied are above the beta transus of the material and, therefore, the consolidation of the powder takes place when elemental titanium is characterised by a B.C.C. structure which is more deformable than the H.C.P. structure. From Figure 2, it can also be seen that the advantage of applying a uniaxial pressure is much more important at low sintering temperatures because the final relative density is remarkably higher, more than $9 \%$, but the difference decreases down to less than $3 \%$ with the increment of the temperature.

\subsection{Hardness of sintered materials}

Vickers hardness values measured on the sintered samples are presented in Figure 3 where it can be seen that the hardness of elemental titanium increases with the processing temperature for both $\mathrm{P} \& \mathrm{~S}$ and HP samples. As it can be seen in Figure 3 a), for a specific processing temperature, the HP samples always reach higher hardness in comparison to the $\mathrm{P} \& \mathrm{~S}$ specimens. This behaviour is better explained considering the variation of the hardness with the relative density (Figure $3 \mathrm{~b}$ ) where it can be seen that the lower the residual porosity (or the higher the relative density), the higher the hardness. This is principally due to the influence of the amount and shape of the residual porosity because starting from $94 \%$ of relative density the pore structure of the material is composed by completely isolated pores. Nonetheless, there is a contribution from the oxygen content which, on average, is $0.03 \mathrm{wt}$.\% higher for HP than for $\mathrm{P} \& \mathrm{~S}$ sample. 

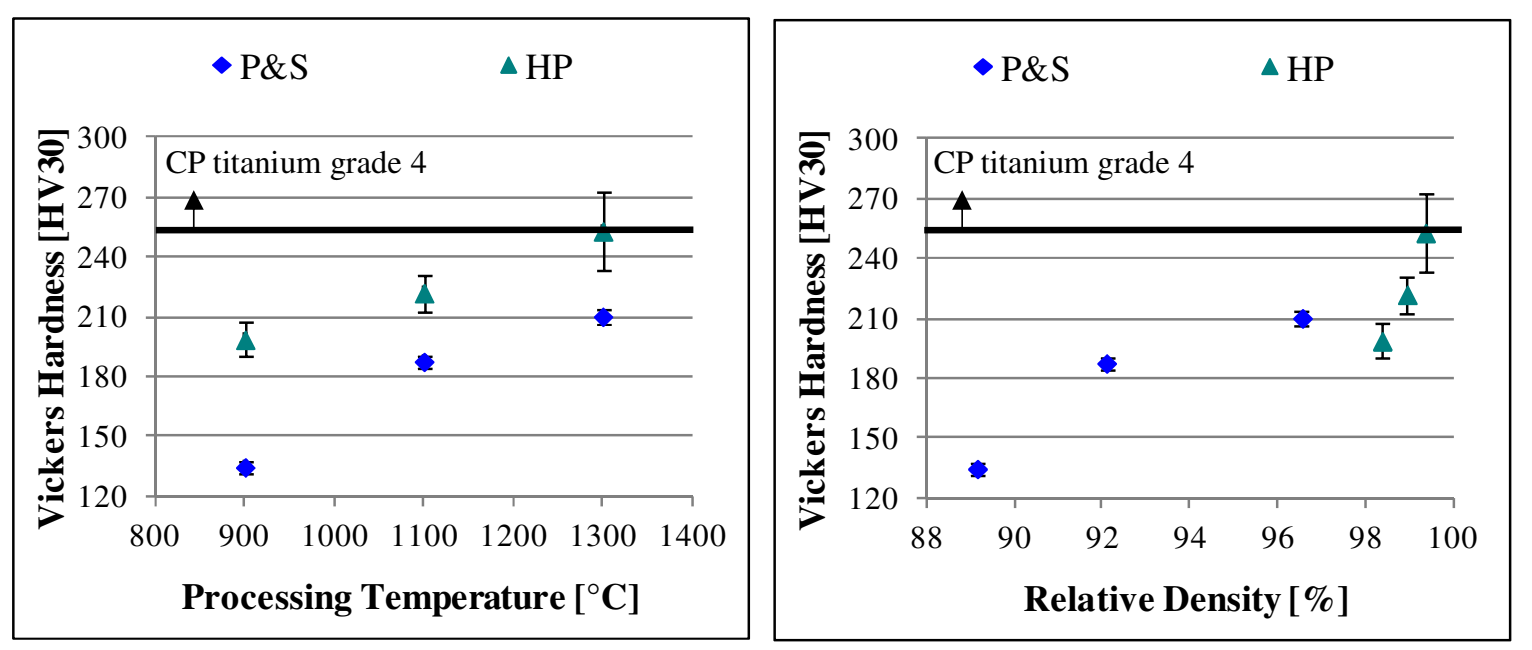

Figure 3. Vickers hardness of P\&S and HP samples as a function of: a) processing temperature and b) relative density.

When it comes to the individual trends of P\&S and HP specimens, the increment in hardness with the processing temperature is due to the combined effect of the increment of the relative density, increase in oxygen content and grain growth. It is worth mentioning that the hardness of the HP samples sintered at the highest temperatures considered is similar to the value of wrought $\mathrm{CP}$ titanium grade 4, whose Vickers hardness is $253 \mathrm{HV}$ [8], because the relative density is more than 99\%. Conversely, P\&S samples show lower hardness due to the presence of the residual porosity (approximately $4 \%$ ).

\subsection{Microstructure of sintered materials}

From the micrographs of P\&S samples shown in Figure 4 , it can be seen that their microstructure is composed by grains of alpha phase and porosity. In particular, from Figure 4 a), at $900^{\circ} \mathrm{C}$ the sintering of the $\mathrm{P} \& \mathrm{~S}$ samples has already started because interparticle necking between the powder particles is clearly visible. Nevertheless, many interparticle boundaries of the former powder particles are still present. On the other side, at $1300^{\circ} \mathrm{C}$ (Figure 4
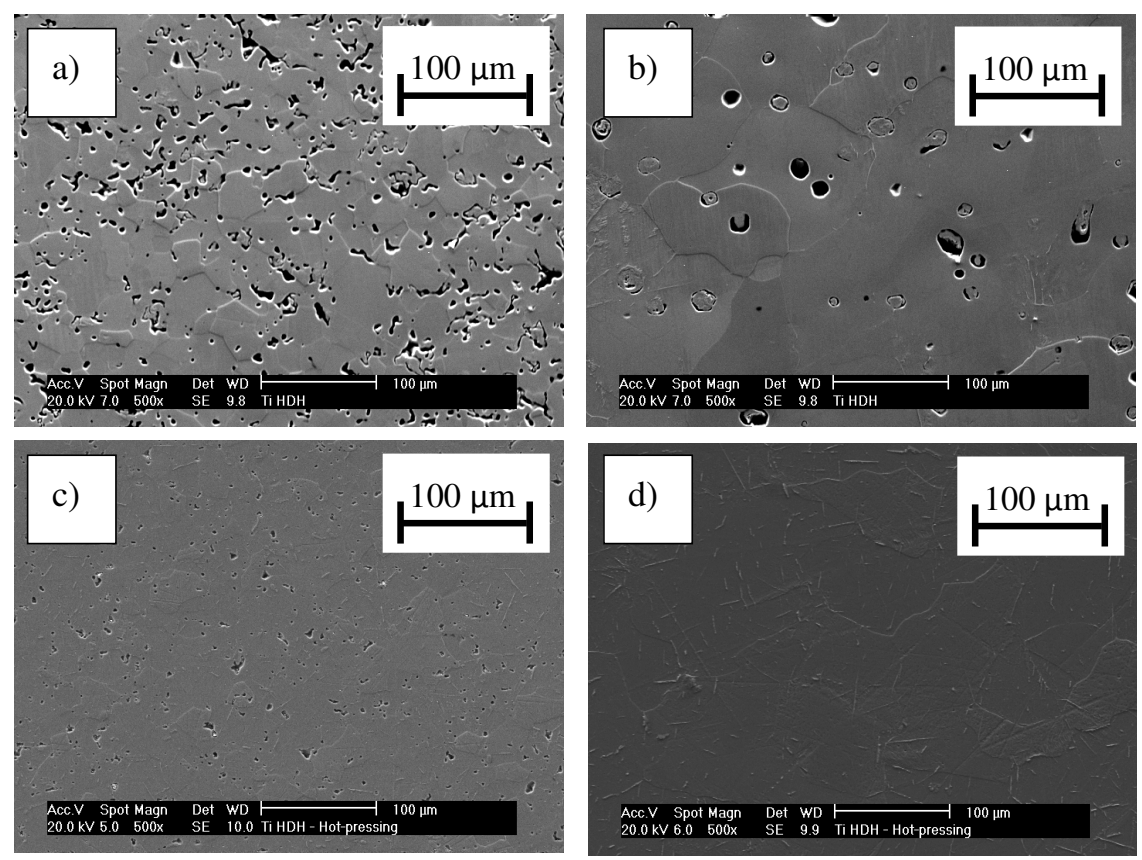

Figure 4. SEM micrographs of $\mathrm{P} \& \mathrm{~S}$ samples sintered at $900^{\circ} \mathrm{C}$ (a) and $1300^{\circ} \mathrm{C} \mathrm{(b)} \mathrm{and} \mathrm{HP}$ samples sintered at $900^{\circ} \mathrm{C}(\mathrm{c})$ and $1300^{\circ} \mathrm{C}(\mathrm{d})$.

b) much of the thermal energy is invested in grain growth and grain rounding. 
The microstructure of elemental titanium processed by means of HP (Figure $4 \mathrm{c}$ and d) is mainly composed by non-equiaxed alpha grains and some transformed beta platelets due to the fact that the material is deformed by applying a uniaxial pressure processed when above the beta transus. Compared to $P \& S$ specimens, the grain size and the amount of porosity of the HP samples are smaller which justifies the higher hardness found (Figure 3).

Elemental titanium reacts with the $\mathrm{BN}$ coating employed to avoid the direct contact between the powder and the graphite tools during consolidation by hot-pressing. This interaction greatly depends on the processing temperature and can be summarised as follows: (1) at $900^{\circ} \mathrm{C}$ there is not a clear visible reaction layer but there is some nitrogen diffusion towards the titanium matrix; (2) at $1100^{\circ} \mathrm{C}$ an irregular layer of approximately $5-10 \mu \mathrm{m}$ in thickness is formed; (3) at $1300^{\circ} \mathrm{C}$ the interaction layer is uniform and almost $20 \mu \mathrm{m}$ in thickness, as it can be seen in Figure 5, which shows a SEM micrograph of the surface of the specimens hot-pressed at $1300^{\circ} \mathrm{C}$.

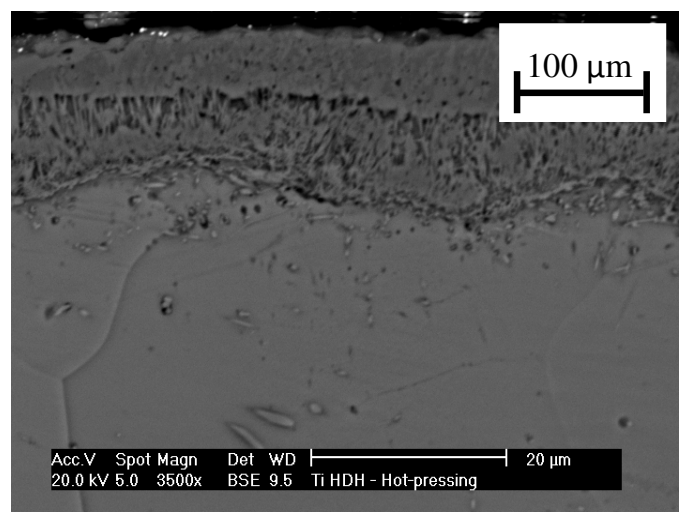

Figure 5. SEM micrograph of the surface of the samples hot-pressed at $1300^{\circ} \mathrm{C}$.

\section{Conclusions}

This study demonstrated that near net-shape, chemically-homogeneous and dense titanium products with properties similar to those of the wrought materials can be produced by means of powder metallurgy techniques which should lower the production costs and, possibly, expands the employment of titanium to new industrial applications.

\section{Acknowledgments}

The authors are thankful for the financial support from the Spanish Ministry of Education through the R\&D MAT2009-14448-C02-02 and MAT2009-14547-C02-02 Projects and from Comunidad de Madrid through the ESTRUMAT (S-2009/MAT-1585) project. The authors would also like to thank Dr. Erik Neubauer from RHP-Technology GmbH and Co. KG for the hot-pressing experiments.

\section{References}

[1] F.H. Froes, D. Eylon, Titanium Powder Metallurgy - A Review, PM Aerospace Materials, Bern Switzerland, 1984, pp. 1-19.

[2] F.H. Froes, D. Eylon, G.E. Eichelman, H.M. Burte, Developments in Titanium Powder Metallurgy, Journal of Metals, 32 (1980) 47-54.

[3] F.H. Froes, D. Eylon, Powder Metallurgy of Titanium Alloys - A Review, Powder Metallurgy International, 17 (1985) 163-167.

[4] G. Friedman, R. Regn, Titanium PM Gyro Components, Metal Powders Report, (1984) 273-281.

[5] R.W. Broomfield, N.G. Turner, B.I. Leat, Application of Advanced Powder Process Technology to Titanium Aeroengine Components, Powder Metallurgy, 28 (1985) 27-34.

[6] C.G. Goetzel, V.S. de Marchi, Electrically Activated Pressure Sintering (Spark Sintering) of Titanium Powders, Powder Metallurgy International, 3 (1971) 80-87.

[7] C.M. WardClose, A.B. Godfrey, S.R. Thompson, Advances in Titanium Alloy Powder, EURO PM 2004, European Powder Metallurgy Association, Viena (Austria), 2004, pp. 261-266.

[8] R. Boyer, G. Welsch, E.W. Collings, Materials Properties Handbook: Titanium Alloys, in: A. International (Ed.), Ohio, USA, 1998. 